\title{
Non-destructive Damage Detection in Fibre Metal Laminates
}

\author{
Patryk Jakubczak $^{1}$ [D Jarosław Bienias ${ }^{1}$ (D)
}

Received: 2 February 2018 / Accepted: 4 April 2019 / Published online: 12 April 2019

(c) The Author(s) 2019

\begin{abstract}
The work explores the possibilities of application of ultrasonic testing in the assessment of fibre metal laminates. Basic problems concerning the use of ultrasonic methods in the research of laminates are explained, and methods for solving these problems are suggested. Tests were conducted using two phased array methods: ultrasonic pulse-echo and through transmission. The efficiency of both selected ultrasonic methods are compared with respect to detecting and dimensioning defects in laminate structures. Based on the analyses and proposed solutions, it has been proven that the developed through transmission phased array (TTPA) method ensures a much more effective, in terms of quality and quantity, assessment of the condition of hybrid laminates than conventional ultrasonic methods, irrespective of the degree of surface deformation and the type of laminate structure.
\end{abstract}

Keywords NDT $\cdot$ Ultrasonic method $\cdot$ Phased array $\cdot$ FML $\cdot$ Fibre metal laminates

\section{Introduction}

Fibre metal laminates (FMLs) are widely used in the aircraft industry, e.g. in large fuselage elements of Airbus A380 [1, 2], due to their high static and fatigue strength along with high resistance to impact and corrosion. Potential errors in their production and use can cause hidden or hardly noticeable damage in the structure of these composite materials, which may lead to significant deterioration of their strength parameters [3, 4]. This means that structures made of FMLs should be tested by non-destructive methods, during both their production and, cyclically, their use.

Non-destructive methods are best suited for quality inspection and further examination of progressing degradation of composite materials. They comprise, among others, ultrasonic methods, thermography and computed tomography [5-10].

The assessment of damage and their extent in FMLs has become an important research subject [11, 12]. Difficulties with signal identification from the opposite side of tested elements resulting from multiple secondary reflections and

Patryk Jakubczak

p.jakubczak@pollub.pl

1 Department of Materials Engineering, Faculty of Mechanical Engineering, Lublin University of Technology,

Nadbystrzycka 36, 20-618 Lublin, Poland structural noises characteristic of composite materials may lead to significant errors in interpretation of ultrasonic testing results. Performing calibration on patterns and signal selection based on the knowledge of laminate structures is one of the methods that makes FML testing more effective. However, the presence of additional, confounding factors such as curvatures and irregular surfaces may prove decisive for test failure.

The fundamental problems in NDT FML testing are associated with the laminar nature of these materials, differences in the acoustic impedance of respective layers and the shape of tested elements, including particularly problematic permanent deformation areas, e.g. after impact. With methods such as thermography, eddy currents or methods utilising $\mathrm{X}$-rays, it impossible to perform correct analyses, including even simplified imaging, due to the presence of metal layers. Therefore, ultrasonic methods are believed to be the fundamental non-destructive FML diagnostic method. Traditional ultrasonic testing utilising the single transducer method presents no possibility for accurate measurements of obtained results due to multiplied reflection signals of respective layers and the problems concerning local deformation and contact loss between the head and the material.

Bisle et al. [11] presented UT methods for Glare inspection. They concluded that, in general, ultrasonic inspection methods turned out to be feasible NDI methods applicable to the inspection of GLARE ${ }^{\circledR}$ parts for in-production and 
in-service tasks. Phased array inspection yields a very clear and detailed image of the inspected area, provided that the instrument is calibrated according to specifications. However, the authors show only a selection of cases which cannot resolve the real problem in FMLs.

Singke [12] presented the results of research on NDT of FMLs. The author concluded that any comparison of ultrasonic attenuation between different configurations is often indirect, and sometimes it is even difficult to compare identical configurations. Small changes in layer thickness can give significant attenuation differences, especially around the resonance points. However, the author has shown that the ultrasonic C-scan through transmission technique is very effective for inspecting FML. The disbond-type discontinuities are very good reflectors of ultrasound. Depth information is not provided by this non-destructive method.

The literature survey has revealed that the problem of non-destructive testing of FMLs has not been thoroughly investigated, and NDT techniques are constantly being improved due to new trends in the development of metal fibre laminates.

Non-destructive assessment of composite materials used in aviation, including FMLs, should encompass the aspects of defect location, shape, dimensions and depth, which is not possible with every method. As regards the in-service damage of composite materials and FMLs, defects take various forms depending on the dominant mechanism of degradation, e.g. they may propagate between different layers, depending on the laminate structure. One of the most significant types of load exerting a negative effect on laminate structures are impact loads. Based on a comprehensive literature survey, it can be concluded that fibre metal laminate damage includes matrix cracking, delamination between individual composite layers and at the metal-composite interface [13-16].

The purpose of this study is to perform quantitative and qualitative non-destructive testing of polymer-metal hybrid materials under dynamic loads using a new effective method. The proposed novel NDT method for quality control of polymer-metal hybrids is compared with the traditional widely used pulse-echo ultrasonic method and verified by quantitative scanning microscopy analysis of the laminate cross-section.

\section{Materials and Methods}

\subsection{Materials}

Two types of fibre metal laminates were non-destructive tested: aluminium/glass fibre (AGL) and titanium/carbon fibre (HTCL). For AGL laminates, a glass fibre-reinforced polymer (UD prepreg, $0.25 \mathrm{~mm}$ thick, Hexcel, USA) was used. For HTCL laminates, carbon fibres (UD prepreg $0.125 \mathrm{~mm}$, Hexcel, USA) were used. Table 1 lists the laminates used in ultrasonic tests.

HTCLs of various structures were used to develop and verify the proposed method for estimating elastic wave propagation speed. On the other hand, AGLs were used to specify attenuation based on the influence of respective laminate layers and to specify the damage area and width in the laminate after impact by pulse-echo and through transmission methods.

AGL and HTCL laminates were manufactured by the autoclave method (Scholz Maschinenbau, Germany) at the Department of Materials Engineering at the Lublin University of Technology.

\subsection{Methods}

Two different non-destructive methods were used for AGL damage evaluation: the traditional ultrasonic pulse-echo (UT) method and a novel through transmission method, both used in phased array (TTPA) mode. In order to verify

Table 1 Fibre metal laminates used in ultrasonic tests

\begin{tabular}{|c|c|c|c|c|c|c|}
\hline No. & Material & Configuration & $\begin{array}{l}\text { Metal layer thick- } \\
\text { ness (mm) }\end{array}$ & $\begin{array}{l}\text { Total laminate } \\
\text { thickness }(\mathrm{mm})\end{array}$ & MVF & Comments \\
\hline 1. & HTCL & $\begin{array}{l}2 / 1 \\
\mathrm{Ti} /(0 / 90)_{6} / \mathrm{Ti}\end{array}$ & 0.5 & 2.5 & 0.4 & Flat samples \\
\hline 2. & & $\begin{array}{l}3 / 2 \\
\mathrm{Ti} /(0 / 90)_{2} / \mathrm{Ti} /(90 / 0)_{2} / \mathrm{Ti}\end{array}$ & 0.5 & 2.5 & 0.6 & Flat samples \\
\hline 3. & & $\begin{array}{l}3 / 2 \\
\mathrm{Ti} /(0 / 90 / 0)_{2} / \mathrm{Ti} /(0 / 90 / 0)_{2} / \mathrm{Ti}\end{array}$ & 0.3 & 2.5 & 0.36 & Flat samples \\
\hline 4. & & $\begin{array}{l}4 / 3 \\
\mathrm{Ti} /(0 / 90 / 0) / \mathrm{Ti} /(0 / 90 / 90 / 0) / \mathrm{Ti} / \\
\quad(0 / 90 / 0) / \mathrm{Ti}\end{array}$ & 0.3 & 2.5 & 0.48 & Flat samples \\
\hline 5. & AGL & $\begin{array}{l}2 / 1 \\
\mathrm{Al} /(0 / 90)_{2} / \mathrm{Al}\end{array}$ & 0.5 & 1.5 & 0.66 & $\begin{array}{l}\text { Flat and impacted samples } \\
\text { (locally deformed and } \\
\text { delaminated) }\end{array}$ \\
\hline
\end{tabular}


defects identified in FMLs with non-destructive methods, a scanning microscopy-based method for analysing image at cross-sections was used.

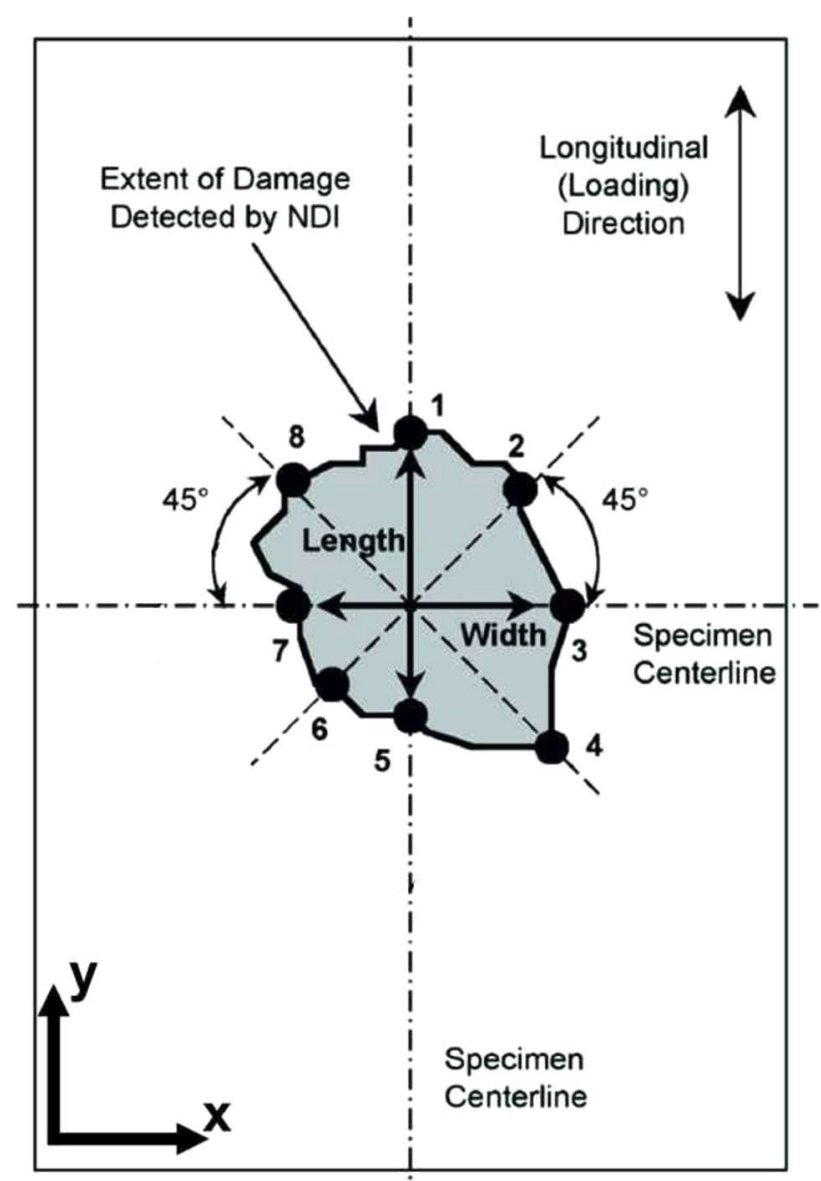

Fig. 1 Measurement of extent of damage according to ASTM 7136 standard
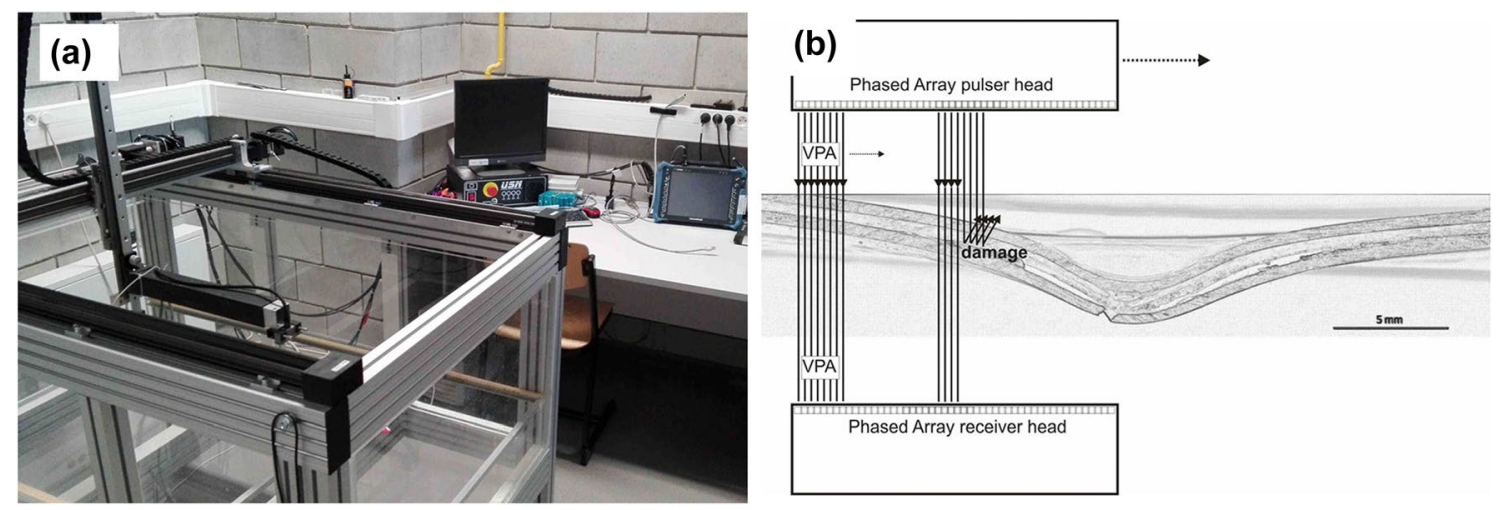

\subsubsection{Traditional Pulse-Echo Ultrasonic Method (UT)}

The pulse-echo ultrasonic (UT) method is widely used in composite material and aircraft structures testing.

The tests were performed with the use of the OmniScan MXU-M ultrasonic defectoscope (Olympus, Japan), equipped with a monotransducer head with a frequency of $5 \mathrm{MHz}$ (Olympus V201-RM, Japan). The head used the delay line (10.15 $\mu$ s wedge delay). Tests were performed in a 4-10 dB amplification range.

The AGL after low speed impact (impact tower, Instron 9340) within the energy range of 2.5-20 J was subjected to full surface scanning by identifying eight points in the area of potential structure defects (Fig. 1). The ASTM D7136 standard guide of damage evaluation after impact is presented in Fig. 1.

\subsubsection{Through Transmission Phased Array}

The other method required the use of a custom-designed stand for through transmission phased array (TTPA) scanning. Figure 2 presents the test stand and a schematic drawing of defects in the FML structure.

The TTPA analyses made use of the OmniScan MXII ultrasonic defectoscope (Olympus, Japan) and TomoView Inspection software for results analysis (Olympus, Japan). The TTPA tests also utilised the Olympus 5L64-I1 ultrasonic transducer (64 piezoelements, frequency $5 \mathrm{MHz}$, elevation $10.0 \mathrm{~mm}$, active aperture $38.4 \mathrm{~mm}$, pitch $0.6 \mathrm{~mm}$ ), virtual aperture VPA ( 8 piezoelements, wave angle: $0^{\circ}$ ), and Olympus 5L64-I1 receiver transducer (64 piezoelements, frequency $5 \mathrm{MHz}$, elevation $10.0 \mathrm{~mm}$, active aperture $38.4 \mathrm{~mm}$, pitch $0.6 \mathrm{~mm}$ ), virtual aperture VPA ( 8 piezoelements, wave angle: $0^{\circ}$ ). The kinetic system of the head was developed based on a 3-axis movement controller of transducers, USN 3D6A (Poland), Mach3 software. The tests were conducted in a $10-18 \mathrm{~dB}$ amplification range.

Fig. 2 TTPA test stand (a) and schematic design (b) of FML damage assessment 


\subsubsection{Scanning Electron Microscope Analysis (SEM)}

Results of the non-destructive tests, i.e. laminate internal structure analysis, were verified with the use of a scanning electron microscope (NovaNano SEM 450, FEI, Japan). After the ultrasonic tests, dynamically loaded samples were coated with polymer resin, precisely cut, ground and buffed to achieve the central point of impactor influence (cross-section). The total length of individual delamination was determined based on a SEM image of damaged laminate structure. Figure 3 shows an example of the cross-section of AGL subjected to dynamic loads and SEM images of the microstructure at the ends and in the central part of delamination between composite layers with the reinforcing fibre orientation (0/90).

\section{Results and Discussion}

\subsection{The Velocity of Longitudinal Wave in Fibre Metal Laminates}

Fibre metal laminates are characterised by their layered nature and various acoustic impedance values of individual layers. This causes interferences and poses significant problems with interpretation of non-destructive test results [12]. The number of layers with various acoustic properties affecting the conditions of longitudinal wave propagation speed in FMLs is primarily associated with metal volume fraction. Analysing only this part of wave which penetrates all consecutive layers of the laminate, one can assume that it propagates through the laminate with different speeds in individual layers. The theoretical, simplified longitudinal elastic wave propagation speed was defined using metal volume fraction (MVF) (Eq. 1). For FMLs, properties based on MVF can be described according to relation (2) [17].

$\mathrm{MVF}=\frac{\sum_{1}^{p} t_{\mathrm{metal}}}{t_{\mathrm{FML}}}$ where $\mathrm{p}$-number of metal layers; $\mathrm{t}_{\text {metal }}$-thickness of metal $[\mathrm{mm}] ; \mathrm{t}_{\mathrm{FML}}$ - total thickness of laminate.

FML property $=\mathrm{MVF} \times$ metal layer property

$$
+(1-\mathrm{MVF}) \times \text { composite layer property }
$$

It was assumed that the propagation speed of an elastic wave travelling perpendicular to the surface of the individual layers could be determined by the rule of mixtures, on the following assumptions: there were no disturbances connected with the actual contact surface; the anodic layer used on the metal surface in fibre metal laminates did not influence penetration and reflection; the layers were perfectly parallel with one another and perpendicular to the incident wave. Taking into account the above, for fibre metal laminates, depending on their design (number and thickness of individual layers), the resultant theoretical longitudinal wave propagation speed can be determined based on relation (3):

$v_{\mathrm{FML}}=\left(\frac{\sum_{1}^{p} t_{\text {metal }}}{t_{\mathrm{FML}}}\right) \times v_{\text {metal }}+\left(1-\frac{\sum_{1}^{p} t_{\text {metal }}}{t_{\mathrm{FML}}}\right) \times v_{\text {comp }}$

where $\nu_{\mathrm{FML}}$-longitudinal wave propagation speed in FML $(\mathrm{m} / \mathrm{s}) ; \nu_{\mathrm{FML}}$ - longitudinal wave propagation speed in metal $[\mathrm{m} / \mathrm{s}] ; \nu_{\mathrm{FML}}$ - longitudinal wave propagation speed in composite material $[\mathrm{m} / \mathrm{s}]$.

Longitudinal wave propagation speeds in the tested HTCLs were determined by experimental $\left(\nu_{\text {exp }}\right)$ and analytical $\left(\nu_{\text {theor }}\right)$ means.

On the basis of the results listed in Table 2, it was determined that with the laminate design and type of individual components being known, it is possible to determine the theoretical propagation speed of an elastic wave in the FML, which (as specified) does not deviate from the actual value by more than $6 \%$, based on the metal volume fraction (MVF). At the same time, it was demonstrated that elastic wave propagation
Fig. 3 Delamination width measurement using scanning electron microscope for laminate subjected to dynamic load with energy of $15 \mathrm{~J}$

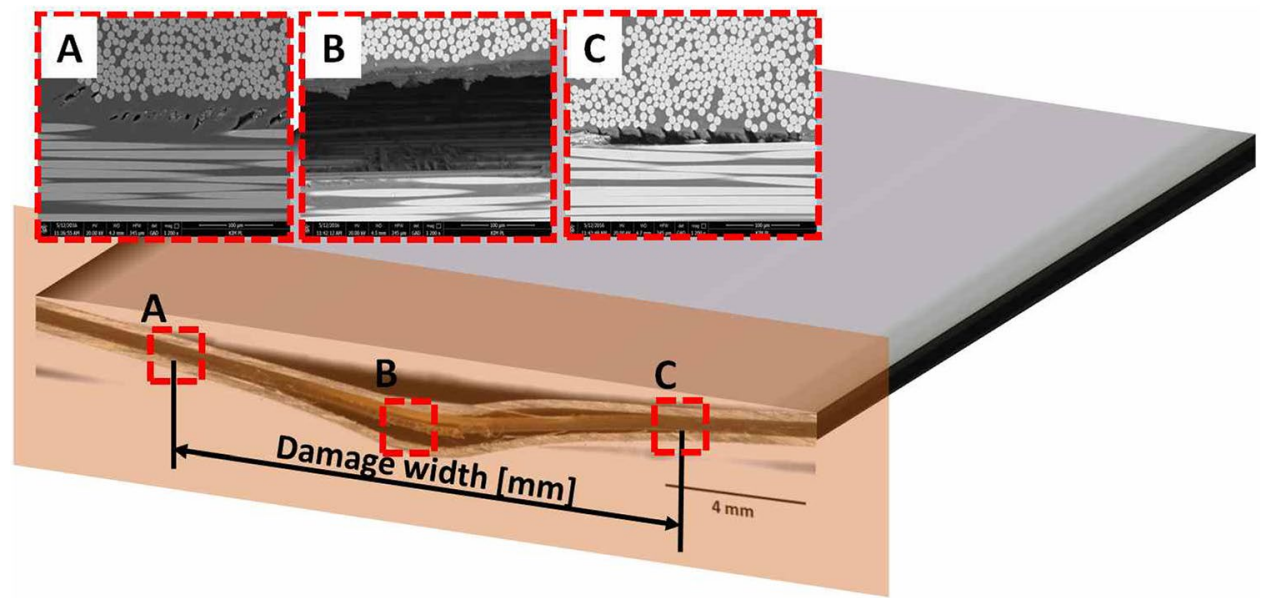


Table 2 Longitudinal wave propagation speeds in HTCLs with various values of metal volume fraction

\begin{tabular}{lllll}
\hline Laminate no. & MVF & $\nu_{\text {exp }}(\mathrm{m} / \mathrm{s})$ & $\nu_{\text {theor }}(\mathrm{m} / \mathrm{s})$ & Mismatch $(\%)$ \\
\hline CFRP & 0 & 3014 & 3100 & 2.80 \\
HTCL no. 1 & 0.4 & 3571 & 3760 & 5.02 \\
HTCL no. 2 & 0.6 & 3880 & 4140 & 6.28 \\
HTCL no. 3 & 0.36 & 3739 & 3684 & 1.47 \\
HTCL no. 4 & 0.48 & 3937 & 3912 & 0.63 \\
Titanium & 1 & 6085 & 6100 & 0.25 \\
\hline
\end{tabular}

speeds in FMLs could be forecast by a linear dependence, without any calculations, with an accuracy of approx. $\pm 10 \%$, based on a nearly linear dependence of wave propagation speed to MVF (Fig. 4).

At the same time, it is worth highlighting that elastic wave propagation speeds in FML materials are associated with a significant degree of uncertainty due to the presence of numerous instances of noise (reflections from individual layers), and hence it is impossible to identify the signal reflected from the opposite laminate surface without an in-depth knowledge of structure of the tested laminate. Consequently, the determination of correct thickness and depth of individual defects in layers of unspecified number and thickness is impossible in practice.

\subsection{Multiphase and Multilayered Nature of Fibre Metal Laminates}

The first, fundamental problem in ultrasonic testing of FMLs concerns the differences in acoustic impedance of individual layers responsible for physical borders of the penetrating mechanical wave, which triggers a series of wave phenomena, including the division of the wave on the border of two media into the reflected (Eq. 4) and penetrating (Eq. 5) parts.

$k_{l}=\frac{I_{\text {reflected }}}{I_{\text {primary }}}=\left(\frac{z_{2}-z_{1}}{z_{2}+z_{1}}\right)^{2}$
$q_{l}=\frac{I_{\text {primary }}}{I_{\text {reflected }}}=\frac{4 z_{1} z_{2}}{\left(z_{1}+z_{2}\right)^{2}}$

where $\mathrm{k}_{1}$ —reflection coefficient; $\mathrm{q}_{1}$ —penetration coefficient; $\mathrm{I}_{\text {reflected }}$-reflected wave intensity; $\mathrm{I}_{\text {primary }}$-(primary) incident wave intensity; $z_{1}$-acoustic wave impedance of the first medium; $\mathrm{z}_{1}=\rho_{1} \mathrm{c}_{1}\left(\mathrm{~g} / \mathrm{cm}^{2} \mathrm{~s}\right) ; \mathrm{z}_{2}$-acoustic wave impedance of the other medium, $z_{2}=\rho_{2} c_{2}\left(g / \mathrm{cm}^{2} s\right) ; \rho$-medium density $\left(\mathrm{g} / \mathrm{cm}^{3}\right) ; \nu$-wave propagation speed in a given medium $(\mathrm{m} / \mathrm{s})$.

For complex (multilayered) materials such as fibre metal laminates, where the number of metal layers is one more than the number of composite layers and the composite layers are within the laminate, it is possible to theoretically estimate the penetration coefficient for the entire laminate $q_{\mathrm{FML}}$ according to Eq. 6 .

$q_{\mathrm{FML}}=\left(\frac{4 z_{1} z_{2}}{\left(z_{1}+z_{2}\right)^{2}}\right)^{n-1} \times\left(\frac{4 z_{2} z_{1}}{\left(z_{2}+z_{1}\right)^{2}}\right)^{m}$

where $\mathrm{n}$-number of metal layers; $\mathrm{m}$-number of composite layers.

Despite the applied simplifications (exclusion of the actual contact surface, damping and wave phenomena) in the theoretical analysis, one should note that in the case of non-destructive tests of 2/1 FMLs by the pulse-echo method, A-scan signals constitute at least several signals of reflections from individual layers. Due to the multilayered nature of FMLs, the signal which reaches the opposite external surface has a greatly reduced amplitude (Fig. 5, point 1b), which results in it being indistinguishable from the signals reflected from other interlayers (Fig. 5, point 1e). In the case of more complex laminates, e.g. $3 / 2,4 / 3$, the number of reflected signals increases proportionately, as the signal reflected from the bottom decreases. The actual A-scan images of 2/1 FML can contain much more noise than shown by the theoretical analysis of the present surfaces of interface with different acoustic impedance. One should note that this is a result, among others, of:
Fig. 4 Longitudinal ultrasonic wave propagation speed in titanium carbon fibre laminates depending on metal volume fraction

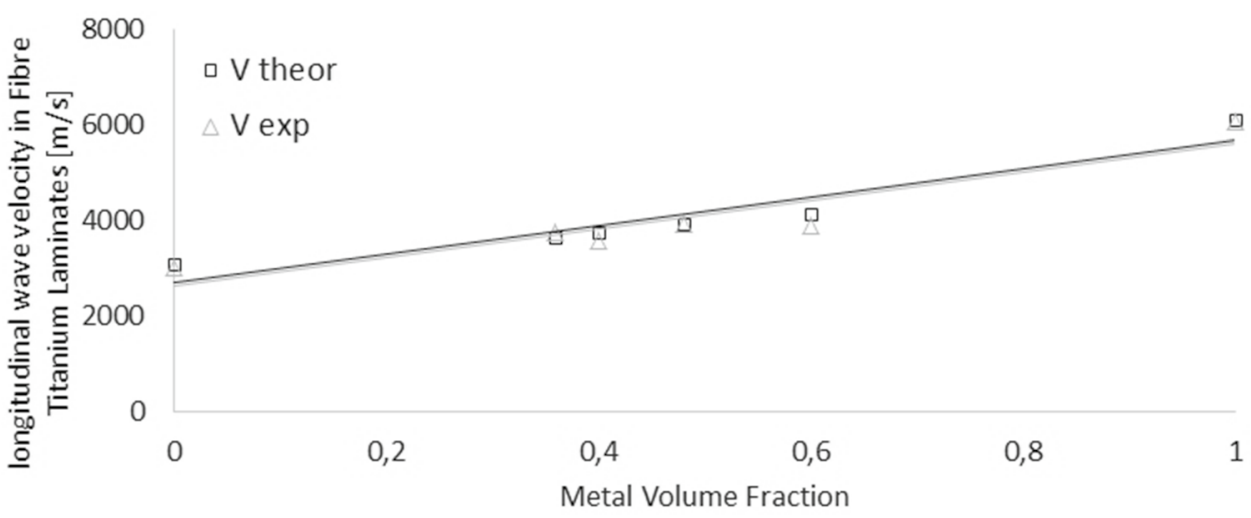


Fig. 5 Simplified model of ultrasonic wave penetration and reflection in AGL laminate

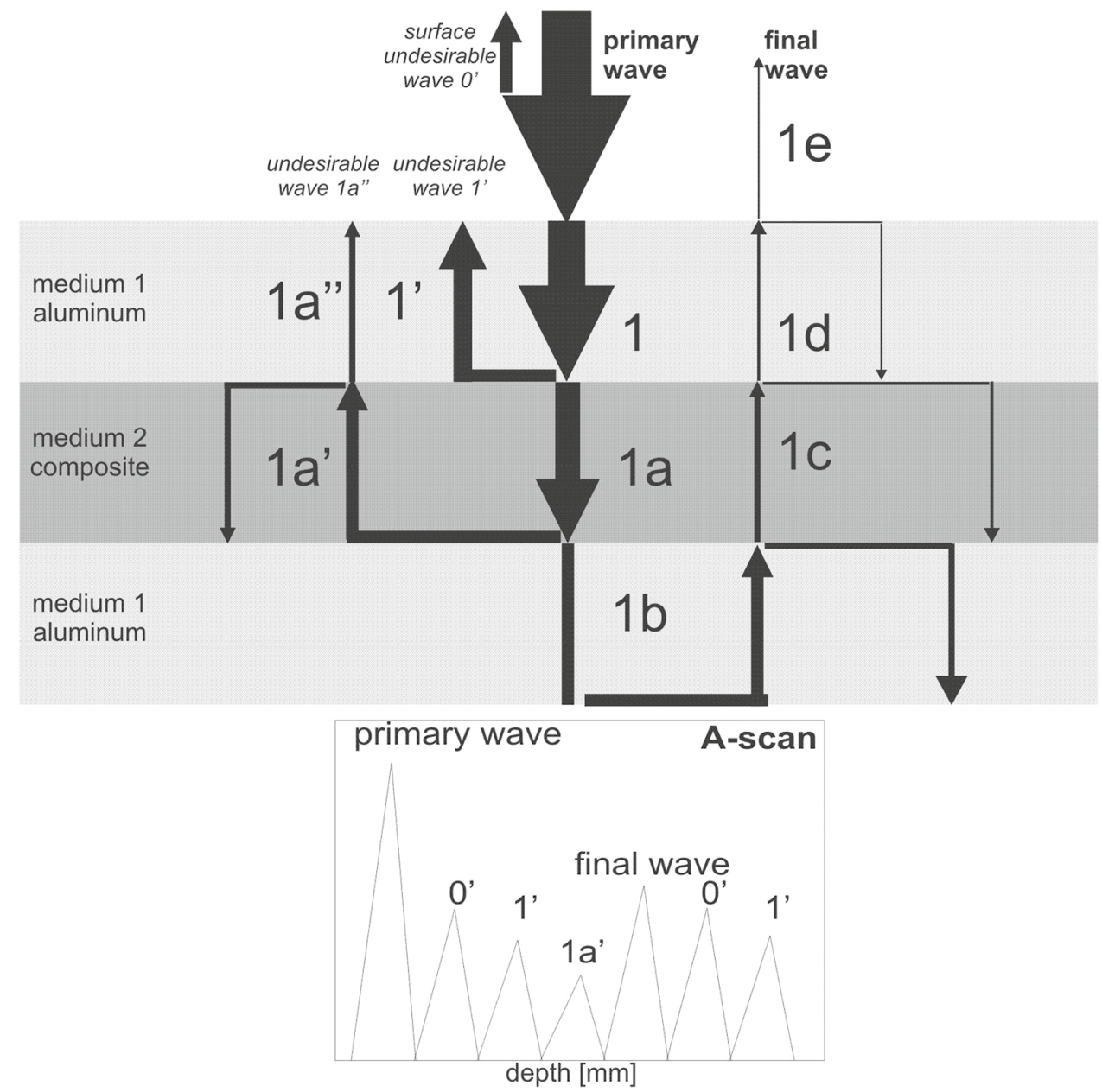

- The morphology of the actual contact surface of two media, for which the disturbed waveform, and thereby the signals registered in the head, generates an additional noise and decreases the amplitude and location of signals at given depths,

- The presence of microroughness or surface curvatures distorts the perpendicularity of wave introduction into the first medium, which can result in, among other things, limited total adherence of the ultrasonic head to the tested surface, generation of additional noise or intensification of other undesired wave phenomena in the laminate (e.g. diffraction).

\subsection{Damage Area Identification in Fibre Metal Laminates After Impact}

\subsubsection{Pulse-Echo (UT) of FML After Impact}

Due to the difficulties connected with non-destructive testing of FMLs by the pulse-echo method, the authors applied an A-scan reference imaging method to determine a defect-free area, and then compared the reference image with A-scans of remaining areas (Fig. 6).

Eight points were specified within the area of the changed waveform. In points 1, 2, 3, 6, 8 (Fig. 6) an additional signal was revealed as well as decreased amplitudes of subsequent secondary signals. In points $4,5,7$, it was possible to observe signal displacement in the path axis and a significant decrease in the amplitude of following signals. Each of the observed signal disruptions was classified as a point indicating a structural defect. On the basis of the above, an outline of the defect was created (Fig. 6). The location of a potential defect in the impact zone (highest deformation) and its shape indicate that the structural defects were detected correctly. The delamination of polymer composite materials under impact propagates in line with the direction of fibres in individual layers, forming the cross section of the defect outline $[18,19]$. This has not yet been confirmed in fibremetal laminates.

Using the pulse-echo method, the area and width of damage in AGLs were determined after a series of impacts with an energy ranging from 2.5 to $20 \mathrm{~J}$ (Table 3 ). 


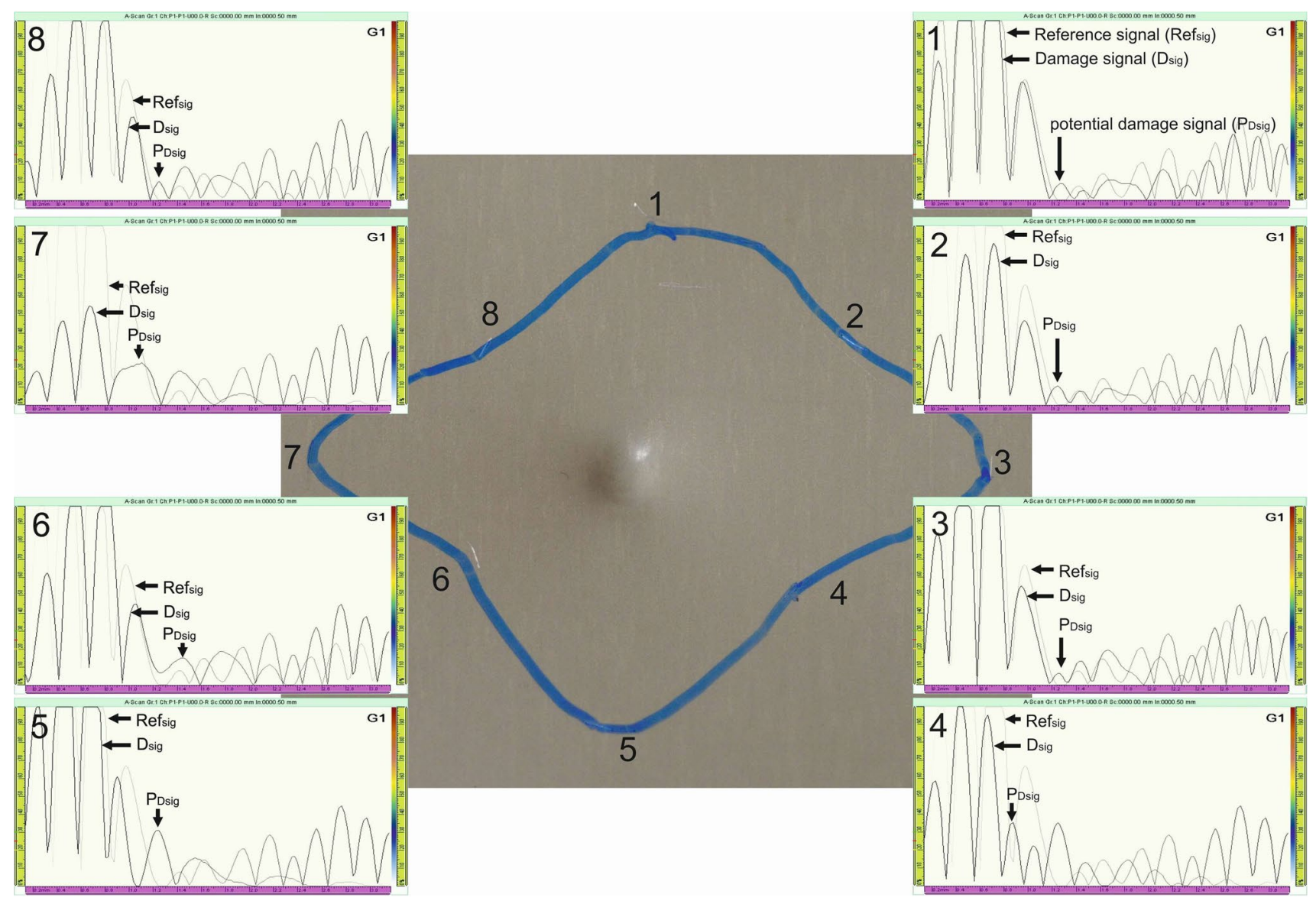

Fig. 6 Example of ultrasonic testing results of AGL after $20 \mathrm{~J}$ impact

Table 3 UT results of damage evaluation in AGL after low-velocity impact

\begin{tabular}{llllll}
\hline Impact energy $(\mathrm{J})$ & 2.5 & 5 & 10 & 15 & 20 \\
\hline Damage area $\left(\mathrm{mm}^{2}\right)$ & 525 & 611 & 1514 & 2512 & 2336 \\
$\begin{array}{c}\text { Damage width along x } \\
\text { axis }(\mathrm{mm})\end{array}$ & 26.3 & 19.4 & 42.5 & 41.1 & 55.6 \\
\hline
\end{tabular}

The conducted non-destructive analysis of AGLs following impact loads within the energy range of 2.5-20 J demonstrates no relationship between damage area increase and impact energy. A similar conclusion can be drawn after analysing the damage width along the adopted $\mathrm{x}$-axis in the centreline of the sample.

\subsubsection{Through Transmission Phased Array Method}

The through transmission phased array (TTPA) method is an alternative to previously used methods for fibre metal laminate evaluation following dynamic loads. In contrast to pulse-echo methods, through transmission methods are not sensitive to the shape and multilayered structure of laminates [12]. They require, however, two-sided access to the tested element. Figure 7 shows examples of the results of tests of AGLs subjected to impacts with an energy of 2.5-15 J, in accordance with the TTPA method.

In the TTPA method, a colour scale illustrates damage easily distinguishable by clear contrast. The clear separation between the areas with different damping properties of the wave passing through the material eliminates the risk of incorrect identification of the damaged and undamaged zones. Results of geometric measurements of AGL laminate damage, as revealed by TTPA testing, are given in Table 4 .

The results show an increase in damage area and width along the $\mathrm{x}$-axis combined with an increase in impact energy. The values of damage area in AGL laminates measured by the UT and TTPA methods are extremely different. Their mismatch is in the range of $440-1675 \%$. 

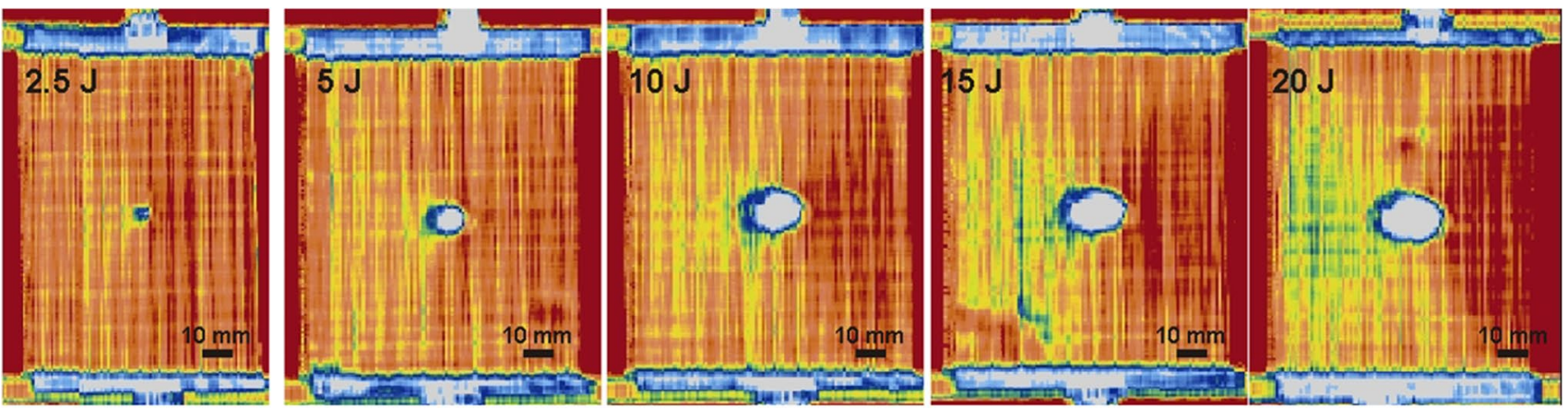

Fig. 7 Damage of ALG after impact identified by TTPA method

Table 4 TTPA results of damage evaluation in AGL after low-velocity impact

\begin{tabular}{lccccc}
\hline Impact energy $(\mathrm{J})$ & 2.5 & 5 & 10 & 15 & 20 \\
\hline Damage area $\left(\mathrm{mm}^{2}\right)$ & 31.2 & 139.9 & 336.5 & 376.6 & 440.5 \\
$\begin{array}{l}\text { Damage width along } \\
\text { x-axis (mm) }\end{array}$ & 7.5 & 15 & 22 & 23 & 27 \\
\hline
\end{tabular}

Table 5 Microscopic results of AGL damage evaluation after lowvelocity impact

\begin{tabular}{llllll}
\hline Impact energy $(\mathrm{J})$ & 2.5 & 5 & 10 & 15 & 20 \\
\hline Damage width $(\mathrm{mm})$ & 8.2 & 16.6 & 22.5 & 23.8 & 28.3 \\
\hline
\end{tabular}

\subsubsection{Verification of Damage Width by Scanning Electron Microscope Analysis}

Microscopic observations of AGL laminates subjected to impact with the energy ranging from 2.5 to $20 \mathrm{~J}$ showed the presence of delamination at the metal-composite phase boundary and between the composite layers $(0 / 90)$. At the same time, it was observed that the most extensive delamination propagation in composite layers was caused by a much lower energy of the composite layer (0/90) interface cracking than in the case of the metal/composite interface [20]. Measured damage widths in AGL laminates along the $\mathrm{x}$-axis are listed in Table 5.

On the basis of delamination width measurements in AGLs, it was determined that, similarly to TTPA nondestructive testing, the total delamination width increases with an increase in impact energy.

Based on total delamination width measurements on the cross section of samples by UT, TTPA and SEM methods, individual methods were compared (Figs. 8, 9).

The distance between the beginning of outmost delamination of FMLs after impact determined with the destructive microscopic method served as a reference for comparison with the results of ultrasonic non-destructive tests (Fig. 8). The discrepancy between the results obtained with the destructive SEM method and the non-destructive TTPA method is not $>10 \%$, whereas with respect to the UT pulseecho method, the discrepancy ranges 16.9-220\% (Fig. 9). These significant differences between the results obtained by the classical ultrasonic testing methods for fibre metal laminates result from the impossibility of separating signals which originate from defects from those reflected from individual laminate layers with various acoustic impedance. The TTPA method is sensitive to secondary reflections, hence the results obtained with this method reflect real condition of the internal structure of laminates, irrespective of design and curvature. Summing up, it has been found that the devised method of scanning in through-transmission mode with the application of phased array is an accurate procedure for quantitative assessment of fibre metal laminate damage, irrespective of the tested object condition (contactless method).

\section{Conclusions}

The work presented the possibilities of applying ultrasonic testing in the assessment of quality of fibre metal laminates. Also, the effectiveness of selected ultrasonic methods in detecting and dimensioning structure defects was compared in qualitative and quantitative terms.

Based on the conducted analyses and proposed solutions, a series of conclusions have been drawn:

1. Based on knowledge of fibre metal laminate structure, design and the type of individual components, it is possible to determine the theoretical propagation speed of an elastic wave in FMLs, based on metal volume fraction.

2. Based on knowledge of laminate design and the type of individual components, it is possible to estimate the wave penetration coefficient for the entire laminate, 

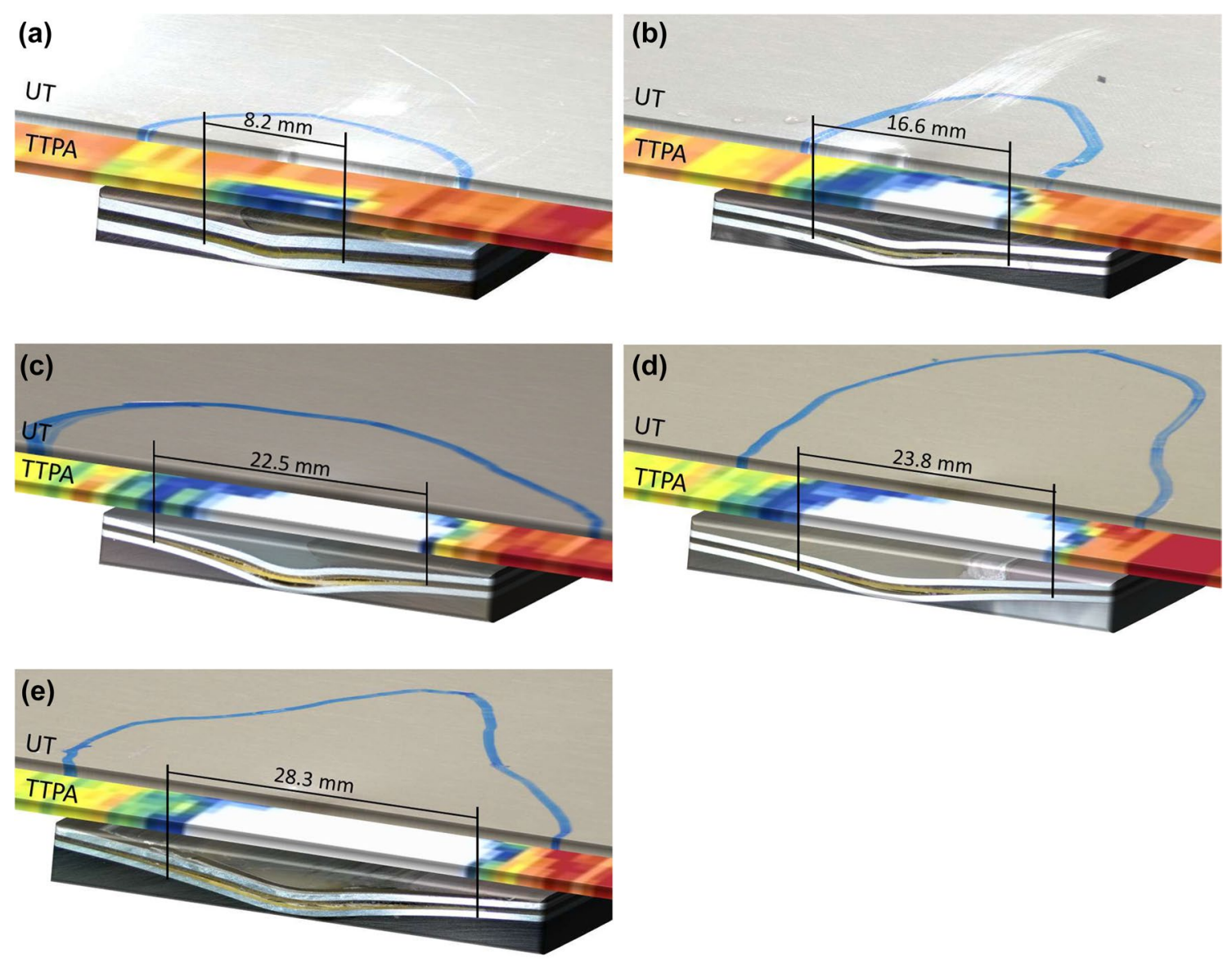

Fig. 8 Comparison of damage width detected in AGL after $2.5 \mathrm{~J}(\mathbf{a}), 5 \mathrm{~J}(\mathbf{b}), 10 \mathrm{~J}(\mathbf{c}), 15 \mathrm{~J}(\mathbf{d})$ and $20 \mathrm{~J}(\mathbf{e})$

Fig. 9 Comparison of damage width in AGL detected by three methods

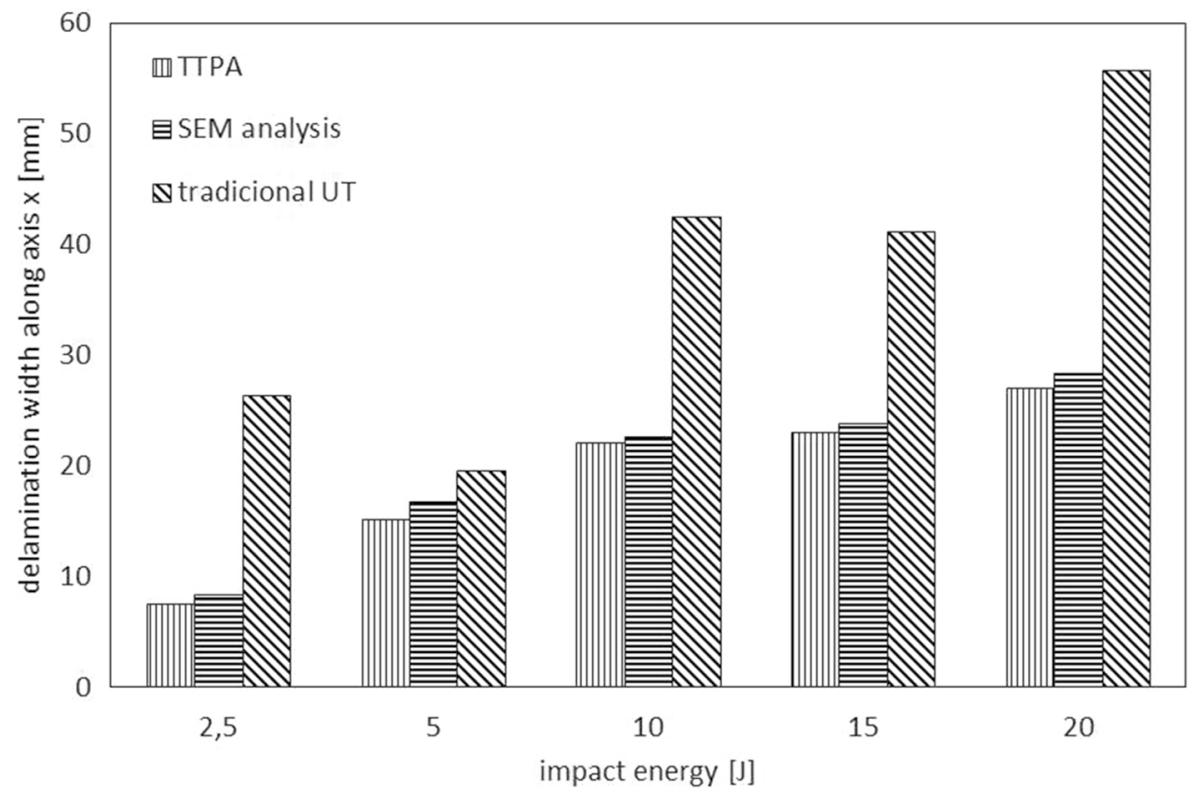


assuming wave impedance coefficients of the medium separately for metal and composite layers.

3. The devised ultrasonic through transmission phased array method facilitates the detection and accurate dimensioning of delamination in fibre metal laminates, irrespective of the laminate type, its design and the presence of potential deformation or other surface defects.

Acknowledgements This research is based on results of a project financed by the National Science Centre, allocated on the basis of Decision Number DEC-2012/05/N/ST8/03788.

Open Access This article is distributed under the terms of the Creative Commons Attribution 4.0 International License (http://creativeco mmons.org/licenses/by/4.0/), which permits unrestricted use, distribution, and reproduction in any medium, provided you give appropriate credit to the original author(s) and the source, provide a link to the Creative Commons license, and indicate if changes were made.

\section{References}

1. Wu, G., Yang, J.M.: The mechanical behavior of GLARE laminates for aircraft structures. JOM 57, 72 (2005)

2. Vogelesang, L.B., Vlot, A.: Development of fibre metal laminates for advanced aerospace structures. J. Mater. Process. Technol. 103, 1-5 (2000)

3. Laliberte, J.F., Poon, C., Straznicky, P.V., Fahr, A.: Post-impact fatigue damage growth in fiber-metal laminates. Int. J. Fatigue 24, 249-256 (2002)

4. Papanicolaou, G.C., Stavropoulos, C.D.: New approach for residual compressive strength prediction of impacted CFRP laminates. Composites 26, 517-523 (1995)

5. Smith, R.A.: Composite defects and their detection. Mater. Sci. Eng. 3, 103-143 (2003)

6. Li S, Chu TP. Ultrasonic 3D reconstruction of CFRP panel delaminations. In: Fall Conference \& Quality Testing Show 2012, Orlando, FL
7. Schilling, P.J., Karedla, B.R., Tatiparthi, A.K., Verges, M.A., Herrington, P.D.: X-ray computed microtomography of internal damage in fiber reinforced polymer matrix composites. Compos. Sci. Technol. 65, 2071-2078 (2005)

8. Bienias, J., Jakubczak, P., Majerski, K., Ostapiuk, M., Surowska, B.: Methods of ultrasonic testing, as an effective way of estimating durability and diagnosing operational capability of composite laminates used in aerospace industry. Eksploat I NiezawodnMaint. Reliab. 15, 284-289 (2013)

9. Bossi, R.H., Georgeson, G.E.: Composite structure development decisions using X-ray CT measurements. Mater. Eval. 53(10), 203-1198 (1995)

10. Kroeger, T.: Thermographic inspection of composites. Reinf. Plast. 58(4), 42-43 (2014)

11. Bsile W, Meier T, Mueller S, Rueckert S. In-service inspection concept for GLARE ${ }^{\circledR}$ —an example for the use of new UT array inspection systems, ECNDT 2006, Berlin

12. Sinke, J.: Some inspection methods for quality control and inservice inspection of GLARE. Appl. Compos. Mater. 10, 277-291 (2003)

13. Bienias, J., Jakubczak, P.: Impact damage growth in carbon fibre aluminium laminates. Compos. Struct. 172, 147-154 (2017)

14. Bienias, J., Jakubczak, P., Surowska, B., Dragan, K.: Low-energy impact behaviour and damage characterization of carbon fibre reinforced polymer and aluminium hybrid laminates. Arch. Civ. Mech. Eng. 4(15), 925-932 (2015)

15. Bienias, J., Jakubczak, P., Dadej, K.: Low-velocity impact resistance of aluminium glass laminates - experimental and numerical investigation. Compos. Struct. 152, 339-348 (2016)

16. Vogelesang, L.B., Vlot, A.: Development of fibre metal laminates for advanced aerospace structures. J. Mater. Process. Technol. 103, 1-5 (2000)

17. Vlot, A., Gunnink, J.W.: Fiber metal laminates: an introduction. Kluwer Academic Publishers, Dordrecht (2001)

18. Shyr, T.W., Pan, Y.-H.: Impact resistance and damage characteristics of composite laminates. Compos. Struct. 62, 193-203 (2003)

19. Aktas, M., Atas, C., Icnte, M.B., Karakuzu, R.: An experimental investigation of the impact response of composite laminates. Compos. Struct. 87, 307-313 (2009)

20. Bienias, J., Dadej, K., Surowska, B.: Interlaminar fracture toughness of glass and carbon reinforced multidirectional fiber metal laminates. Eng. Fract. Mech. 175, 127-145 (2017) 